\title{
Eccentric Dipoles and Spherical Harmonic Analysis
}

\author{
L. R. Alldredge \\ U.S. Geological Survey, Denver, Colorado, U.S.A. \\ (Received November 2, 1981)
}

Louis HURWITz (1960) wrote a short paper with this same title giving formulas for the potential of eccentric dipoles in both closed form and in terms of spherical harmonic (S.H.) expressions for internal sources. Over the years I have found these expressions very helpful in doing model work.

Recently more attention is being given to geomagnetic models which include external sources. Believing that the S.H. expression for external eccentric dipoles may be useful in such work, I take this occasion to extend the work of Hurwitz to external dipoles.

Consider an eccentric dipole distant $r_{0}$ from the center of a spherical Earth, in colatitude $\theta_{0}$ and east longitude $\lambda_{0}$. Its components of magnetic moment $M_{r}, M_{\theta}, M_{\lambda}$, in the directions of increasing $r_{0}, \theta_{0}$, and $\lambda_{0}$ produce corresponding potentials $W_{r}, W_{\theta}, W_{\lambda}$ at the field point $r, \theta, \lambda$. In a form slightly modified from that given by HuRwITz (1960), which is suggestive as to how the S.H. expression may be derived, the closed form of the potentials in cgs units (as used by Hurwitz) are:

$$
\begin{aligned}
& W_{r}=M_{r} r\left(q-\frac{r_{0}}{r}\right) / \rho^{3}, \\
& W_{\theta}=M_{\theta} r\left(\frac{\partial q}{\partial \theta_{0}}\right) / \rho^{3},
\end{aligned}
$$

and

$$
W_{\lambda}=M_{\lambda} r\left(\frac{\partial q}{\partial \lambda_{0}}\right) / \rho^{3} \sin \theta_{0},
$$

where

$$
\rho^{2}=r^{2}+r_{0}^{2}-2 r r_{0} q
$$

and

$$
q=\cos \theta_{0} \cos \theta+\sin \theta_{0} \sin \theta \cos \left(\lambda-\lambda_{0}\right) .
$$

For internal sources $r>r_{0}$ and each of these can be expressed in the general form

$$
a \sum_{n=0}^{\infty} \sum_{m=0}^{n}(a / r)^{n+1}\left(A_{n_{i}}^{m} \cos m \lambda+B_{n_{i}}^{m} \sin m \lambda\right) P_{n}{ }^{m} .
$$

Hurwitz showed that for $W_{r}$ : 


$$
\begin{aligned}
A_{n_{i}}^{m} & =\left(M_{r} / a^{3}\right)\left(r_{0} / a\right)^{n-1} n Q_{n}{ }^{m} \cos m \lambda_{0} \\
B_{n_{i}}^{m} & =A_{n_{i}}^{m} \tan m \lambda_{0}
\end{aligned}
$$

for $W_{\theta}$ :

$$
\begin{aligned}
& A_{n_{i}}^{m}=\left(M_{\theta} / a^{3}\right)\left(r_{0} / a\right)^{n-1}\left(\mathrm{~d} Q_{n}{ }^{m} / \mathrm{d} \theta_{0}\right) \cos m \lambda_{0} \\
& B_{n_{i}}^{m}=A_{n_{i}}^{m} \tan m \lambda_{0}
\end{aligned}
$$

and for $W_{\lambda}$ :

$$
\begin{aligned}
A_{n_{i}}^{m} & =-\left(M_{\lambda} / a^{3}\right)\left(r_{0} / a\right)^{n-1}\left(m / \sin \theta_{0}\right) Q_{n}{ }^{m} \sin m \lambda_{0} \\
B_{n_{i}}^{m} & =-A_{n}{ }^{m} \cot m \lambda_{0},
\end{aligned}
$$

where $P_{n}{ }^{m}$ and $Q_{n}{ }^{m}$ are Schmidt's partially normalized associated Legendre functions of the polar angles $\theta$ and $\theta_{0}$ respectively and a is an arbitrary constant which can be cancelled out. The constant a is called the radius of the reference sphere, and in practical problems is usually taken to be the radius of the Earth which then makes S.H. coefficients from different analyses intercomparable.

For external sources $r_{0}>r$ and each of the Eqs. (1) can be expressed in the general form

$$
a \sum_{n=0}^{\infty} \sum_{m=0}^{n}(r / a)^{n}\left(A_{n_{e}}^{m} \cos m \lambda+B_{n_{e}}^{m} \sin m \lambda\right) P_{n}{ }^{m} .
$$

It may be shown that the coefficients $A_{n_{e}}^{m}$ and $B_{n_{e}}^{m}$ are for $W_{r}$ :

$$
\begin{aligned}
A_{n_{e}}^{m} & =-\left(M_{r} / a^{3}\right)\left(a / r_{0}\right)^{n+2}(n+1) Q_{n}{ }^{m} \cos m \lambda_{0} \\
B_{n_{e}}^{m} & =A_{n_{e}}^{m} \tan m \lambda_{0}
\end{aligned}
$$

for $W_{\theta}$ :

$$
\begin{aligned}
& A_{n_{e}}^{m}=\left(M_{\theta} / a^{3}\right)\left(a / r_{0}\right)^{n+2}\left(\mathrm{~d} Q_{n}{ }^{m} / \mathrm{d} \theta_{0}\right) \cos m \lambda_{0} \\
& B_{n_{e}}^{m}=A_{n_{e}}^{m} \tan m \lambda_{0}
\end{aligned}
$$

and for $W_{\lambda}$ :

$$
\begin{aligned}
& A_{n_{e}}^{m}=-\left(M_{\lambda} / a^{3}\right)\left(a / r_{0}\right)^{n+2}\left(m / \sin \theta_{0}\right) Q_{n}{ }^{m} \sin m \lambda_{0} \\
& B_{n_{e}}^{m}=-A_{n_{e}}^{m} \cot m \lambda_{0} .
\end{aligned}
$$

The coefficients given in (3) and (5) are exact coefficients which might differ greatly from coefficients derived by least squares using sparse data from the dipoles and a truncated series (ALLDREDGE and KAWASAKI, 1981).

It is interesting to note how the corresponding series in (2) and (4) converge for $W_{r}$ for simple zonal examples.

For $M_{r}=1,000, r_{0}=10, \theta_{0}=0$, and $\lambda_{0}=0$, Eq. (1a) gives $W_{r}=1,000$ at $r=11, \theta=0$, $\lambda=0 ;$ and $W_{r}=-1,000$ at $r=9, \theta=0, \lambda=0$.

Using the series (2) and (3a) for the first example we get 


$$
W_{r}=\frac{M_{r}}{r_{0}^{2}} \sum_{n=0}^{\infty}\left(\frac{r_{0}}{r}\right)^{n+1} n .
$$

and using the series (4) and (5a) for the second example we get

$$
W_{r}=-\frac{M_{r}}{r_{0}^{2}} \sum_{n=0}^{\infty}\left(\frac{r}{r_{0}}\right)^{n}(n+1) .
$$

For the two examples the values of the two infinite series in (6) and (7) are, of course, equal in amplitude but, for these examples, (7), for the external source, converges more rapidly than (6), for the internal source, as can be seen in the Table 1 where the cumulative values of (6) and (7) are shown versus $n$.

Table 1. Cumulative values* of Eqs. (6) and (7) versus $n$ for the two examples described in the text.

\begin{tabular}{rrr}
$n$ & Eq. (6) internal source & Eq. (7) external source \\
\hline 0 & 0 & -10 \\
5 & 97 & -150 \\
10 & 264 & -341 \\
20 & 581 & -661 \\
30 & 786 & -844 \\
40 & 898 & -932 \\
50 & 953 & -972 \\
60 & 979 & -989 \\
70 & 991 & -995 \\
80 & 996 & -998 \\
90 & 998 & -999 \\
100 & 999 & -1000 \\
\hline
\end{tabular}

* Calculated to 7 significant figures then rounded off as shown.

There are no simple zonal solutions for the $M_{\theta}$ and $M_{\lambda}$ dipoles which can be used to intercompare the internal expansion with the external expansion. Comparisons were made, however, as a check on the formulations, by using the series to compute the potentials at an exterior point and at an interior point which are symmetric about the dipole so that the potentials are equal except for the sign. As $n$ increases the potentials overshoot the closed form value by nearly five percent and then tend to oscillate as the true value is approached.

To visualize how this information might be applied to external sources which are more realistic than dipoles, consider a current loop which is symmetrical about the spherical coordinate axis at a distance $r_{1}$ from the origin as shown in the Fig. 1. The potential at $P(r, \theta, \lambda)$, an interior point, can be expressed in the form of (4). By using the technique of replacing the loop by a radially magnetized spherical cap bounded by the loop at a distance $r_{1}$ from the center (see Fig. 1) and integrating exactly as was done in an earlier paper (AlLDREDGE, 1980), it may be shown that the potential is zonal and that the constants $A_{n_{e}}^{0}$ in (4) are given by 




Fig. 1. Projection of current loop on a plane perpendicular to the coordinate axis and its equivalent radially magnetized spherical cap.

$$
A_{n_{e}}^{0}=-2\left(M_{r} / a^{3}\right)\left(a / r_{1}\right)^{n+2}\left((n+1) / \sin \theta_{0}\right) \frac{p_{n}{ }^{1}\left(\cos \theta_{0}\right)}{\sqrt{2 n(n+1)}}
$$

for $n>0$, and by

$$
A_{n_{e}}^{0}=-2\left(M_{r} / a^{3}\right)\left(a / r_{1}\right)^{n+2}\left(1-\cos \theta_{0}\right)
$$

for $n=0$, and

$$
B_{n_{e}}^{m}=0 \quad \text { (because the potential is zonal), }
$$

where $M_{r}=I \pi R_{0}^{2}$ and $I$ is the current.

The expression corresponding to (8) for an internal loop, that is where $r>r_{1}$ in the Fig. 1, is (AlldREDGE, 1980)

$$
A_{n_{i}}^{0}=2\left(M_{r} / a^{3}\right)\left(r_{1} / a\right)^{n-1}\left(n / \sin \theta_{0}\right) \frac{P_{n}{ }^{1}\left(\cos \theta_{0}\right)}{\sqrt{2 n(n+1)}} .
$$

\section{REFERENCES}

Alldredge, L. R., Circular current loops, magnetic dipoles and spherical harmonic analyses, J. Geomag. Geoelectr., 32, 357-364, 1980.

Alldredge, L. R. and K. KAWASAKI, Spherical harmonic analysis in the presence of high harmonics, $J$. Geomag. Geoelectr., 33, 503-515, 1981.

Hurwitz, Louis, Eccentric dipoles and spherical harmonic analysis, J. Geophys. Res., 65, 2555-2556, 1960. 